\title{
Deterministic transition of enterotypes shapes the infant gut microbiome at an early age
}

\author{
Liwen Xiao ${ }^{1,2 \dagger}$, Jinfeng Wang ${ }^{1 * \dagger}$, Jiayong Zheng ${ }^{3 \dagger}$, Xiaoqing $\mathrm{Li}^{3}$ and Fangqing Zhao ${ }^{1,2^{*}}$ (D)
}

\author{
*Correspondence: wangjf@biols.ac. \\ cn; zhfq@biols.ac.cn \\ †Liwen Xiao, Jinfeng Wang and \\ Jiayong Zheng contributed equally \\ to this work. \\ ${ }^{1}$ Computational Genomics \\ Laboratory, Beijing Institutes of Life \\ Science, Chinese Academy of \\ Sciences, Beijing 100101, China \\ Full list of author information is \\ available at the end of the article
}

\begin{abstract}
Background: The succession of the gut microbiota during the first few years plays a vital role in human development. We elucidate the characteristics and alternations of the infant gut microbiota to better understand the correlation between infant health and microbiota maturation.

Results: We collect 13,776 fecal samples or datasets from 1956 infants between 1 and 3 years of age, based on multi-population cohorts covering 17 countries. The characteristics of the gut microbiota are analyzed based on enterotype and an ecological model. Clinical information $(n=2287)$ is integrated to understand outcomes of different developmental patterns. Infants whose gut microbiota are dominated by Firmicutes and Bifidobacterium exhibit typical characteristics of early developmental stages, such as unstable community structure and low microbiome maturation, while those driven by Bacteroides and Prevotella are characterized by higher diversity and stronger connections in the gut microbial community. We further reveal a geography-related pattern in global populations. Through ecological modeling and functional analysis, we demonstrate that the transition of the gut microbiota from infants towards adults follows a deterministic pattern; as infants grow up, the dominance of Firmicutes and Bifidobacterium is replaced by that of Bacteroides and Prevotella, along with shifts in specific metabolic pathways.

Conclusions: By leveraging the extremely large datasets and enterotype-based microbiome analysis, we decipher the colonization and transition of the gut microbiota in infants from a new perspective. We further introduce an ecological model to estimate the tendency of enterotype transitions, and demonstrated that the transition of infant gut microbiota was deterministic and predictable.
\end{abstract}

\section{Background}

Dynamics of the gut microbiota during early development not only has a considerable impact on childhood but also influences their health when children grow up [1, 2]. Unlike that in adults, the microbial community in infants presents a less complex and hypervariable pattern, especially during the first few years after birth [3]. The simple composition of the neonatal microbiota facilitated us to elucidate and highlight the

(c) The Author(s). 2021 Open Access This article is licensed under a Creative Commons Attribution 4.0 International License, which permits use, sharing, adaptation, distribution and reproduction in any medium or format, as long as you give appropriate credit to the original author(s) and the source, provide a link to the Creative Commons licence, and indicate if changes were made. The images or other third party material in this article are included in the article's Creative Commons licence, unless indicated otherwise in a credit line to the material. If material is not included in the article's Creative Commons licence and your intended use is not permitted by statutory regulation or exceeds the permitted use, you will need to obtain permission directly from the copyright holder. To view a copy of this licence, visit http://creativecommons.org/licenses/by/4.0/. The Creative Commons Public Domain Dedication waiver (http://creativecommons.org/publicdomain/zero/1.0/) applies to the data made available in this article, unless otherwise stated in a credit line to the data. 
establishment of the symbiotic relationship between the host and microbes. In contrast, the high plasticity and dependence on external environments of the infant microbiota may provide an opportunity to use external intervention during the early stage to improve children's health in the future.

To date, most of the studies on the gut microbiota in early stages of life have examined cross-sectional samples collected from a single time point $[4,5]$, and the dynamics of gut microbiota from newborn to infancy is unclear due to the lack of high temporal resolution data. To overcome this shortcoming, several longitudinal cohorts with large sample sizes were recruited $[3,6]$. According to a recent TEDDY study [3], the gut microbiota of infants may be roughly divided into three phases, namely developmental (3-14 months), transitional (15-30 months), and stable phases ( $\geq 31$ months). However, the transition pattern and its driving forces during this process remain largely unknown. Recently, several studies have attempted to reveal the structure of infant microbiota using the concept of enterotype $[7,8]$. Unlike the three familiar and consistent enterotypes (Bacteroides, Prevotella, and Ruminococcus) in adults, the infant enterotypes classified through each study were different. The difference may be related to the instability and high diversity of the microbiota from newborn to infancy, or it may result from different sampling stages or insufficient data size. In either case, uniform classification and systematic analysis of infant enterotypes are necessary to focus on the colonization and succession of the gut microbiota in early life.

In addition to the development involved in the natural growth cycle, some critical time points or clinical factors may play important roles in shaping the neonatal microbiota. It was reported that the relative abundance of Firmicutes, such as Clostridium, Streptococcus, and Enterococcus, was much higher in infants delivered by cesarean section. In contrast, Bacteroides and Bifidobacterium showed an evident enrichment in the development of infants delivered via the vaginal route [6]. Postnatal factors such as feeding habits and solid food intake are crucial as well [3, 9]. Several studies have reported differences in the gut microbiota of infants among different ethnicities or continents $[5,10]$, which implies that this diversity may derive from early life stages. The integrated analysis of these factors will help to better understand the early development of human microbiota.

Herein, we longitudinally collected fecal samples of neonates from China and further integrated over 10,000 metagenomic or 16S rRNA sequencing datasets of longitudinal fecal samples from 17 countries, spanning the first 3 years of life. Based on the largest population to date, the microbial assembly, succession, and maturation during early life were elucidated. Through the application of ecological models and the Markov chain, the transition rate of the gut microbiota in the early stages was quantified. Our findings provide comprehensive insights into the initial colonization and transition of the human microbiota through the analysis of enterotypes, which may increase our understanding of the microbiota dynamics in early life.

\section{Results}

Population characteristics

We enrolled a cohort of 101 Chinese full-term healthy neonates in this study; collected their fecal samples at birth, 6, 12, or 18 months of age; and then used metagenomic 
shotgun sequencing to investigate their microbial community structures. We also retrieved metagenomic or 16S rRNA sequencing datasets of longitudinal fecal samples of newborns and infants from 19 public cohorts [6, 8-23]. In total, 13,776 fecal microbiome datasets of 1956 infants aged 1-36 months were obtained and analyzed after quality control and batch effect correction and included seven time points on average for each individual. This combined dataset covered seventeen countries of six continents, including Asia, South and North America, Europe, Australia, and Africa (Fig. 1A, Additional file 1: Table S1).

\section{Classification of the infant gut microbiota in early life based on enterotypes}

Enterotypes were classified in the large population of this study $(n=13,776)$ according to previously described methods [24]. The microbial profiling resulted in four enterotypes (Fig. 1B, Additional file 1: Fig. S1 and Additional file 1: Table S2) with the bacterial genera belonging to phylum Firmicutes, Bifidobacterium, Bacteroides, and Prevotella, dominating enterotypes 1 to 4 . These enterotypes clustered stably with

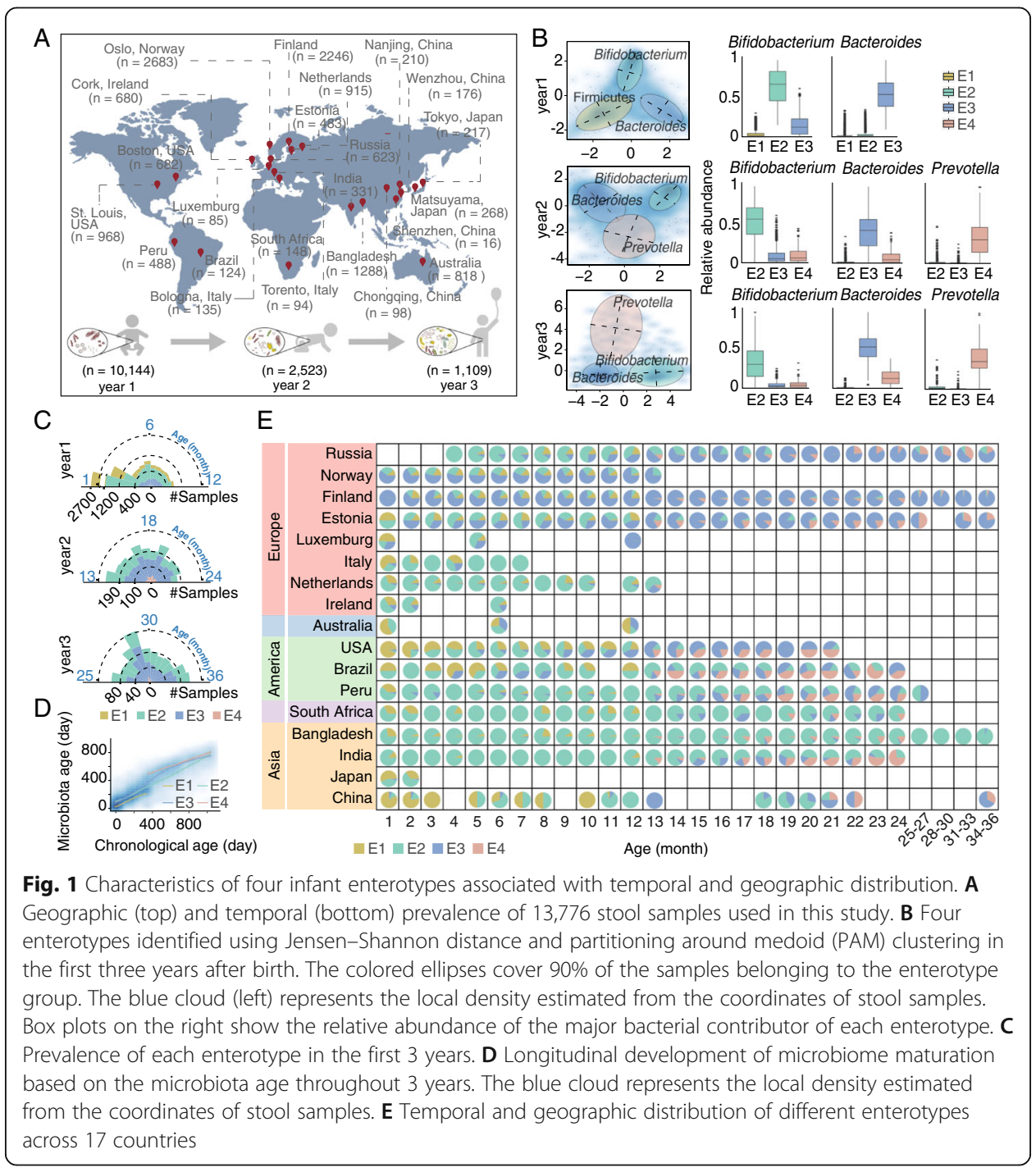


various sample sizes as well as in different datasets (Additional file 1: Figs. S2-4), strongly indicating the reliability of enterotype clustering in infant gut microbiota.

To better understand the four enterotypes and to explore the temporal distributions of the infant microbiota in the first 3 years of life, we identified the emergence windows of each enterotype (Fig. 1B, C and Additional file 1: Fig. S5A-B). For enterotype 1 (E1, $n=3062$ ) dominant bacteria varied at the genus level (Additional file 1: Fig. S5A-B). In contrast, enterotypes $2(\mathrm{E} 2, n=5264)$ and $3(\mathrm{E} 3, n=5052)$, represented by a relatively high abundance of the genera Bifidobacterium and Bacteroides, respectively, were observed constantly in the first 3 years of life. Enterotype $4(E 4, n=398)$ dominated by the anaerobic Prevotella did not appear until the second year of life. Apart from the emergence windows, we also found that the community characteristics varied over time for each enterotype. Although the four enterotypes presented an increased pattern during early development both in diversity and microbiome maturation, E3 and E4 exhibited relatively higher alpha diversity and larger microbiome maturation rate than E1 and E2 after the first year of life (Fig. 1D and Additional file 1: Fig. S5C, Wilcoxon test, $P<0.001$ ), which might represent an advanced developmental stage of the gut microbiota in infants.

To investigate the geographical distribution of the infant microbiota in the first 3 years of life, we subsequently classified the four enterotypes in the populations of different countries. An evident geographical stratification of enterotypes was observed (chisquare test, $P<0.001$ ) (Fig. $1 \mathrm{E}$ and Additional file 1: Fig. S6). For example, among developing countries such as India, Bangladesh, South Africa, Peru, and Brazil, E2 was prevalent throughout the first 3 years of life. In contrast, developed countries in Northern Europe, such as Finland, Norway, and Estonia presented a Bacteroides-predominating E3 for most of the months. Besides these geographical differences, there was a clear trend of enterotype transition over time. For example, in Finland, the existence of E1 and E2 on the early stage was replaced rapidly by the large proportion of E3 with the growth of infants (Fig. 1E), which indicates a strong correlation between developmental stages and transition of enterotypes. Differences in the emergence windows and community characteristics of each enterotype as well as the enterotype preference in diverse countries suggest that the gut microbiota of these infants may be in distinct developmental stages, thereby representing different degrees of maturity.

\section{Distinct enterotypes correspond to different developmental stages of the infant gut microbiota}

To further characterize the gut microbiota in the early stages of life along with the chronological ages and geographical distributions of infants, we compared the relative abundance of gut bacteria at the species or strain levels in four enterotypes based on 1165 metagenomic sequencing profiles from four countries (China, Luxemburg, Italy, and the USA).

As shown in Fig. 2A, the differences in gut microbiota were much greater among enterotypes than among countries, regardless of the infant age (ANOVA test, $R^{2}$ of enterotypes $4.65 \%$ vs $R^{2}$ of nations $2.88 \%, P<0.001$ ). Specifically, no remarkable country-specific species were observed in this study, suggesting that the stratification of enterotypes may not directly result from geographical factors. In contrast, enterotype- 


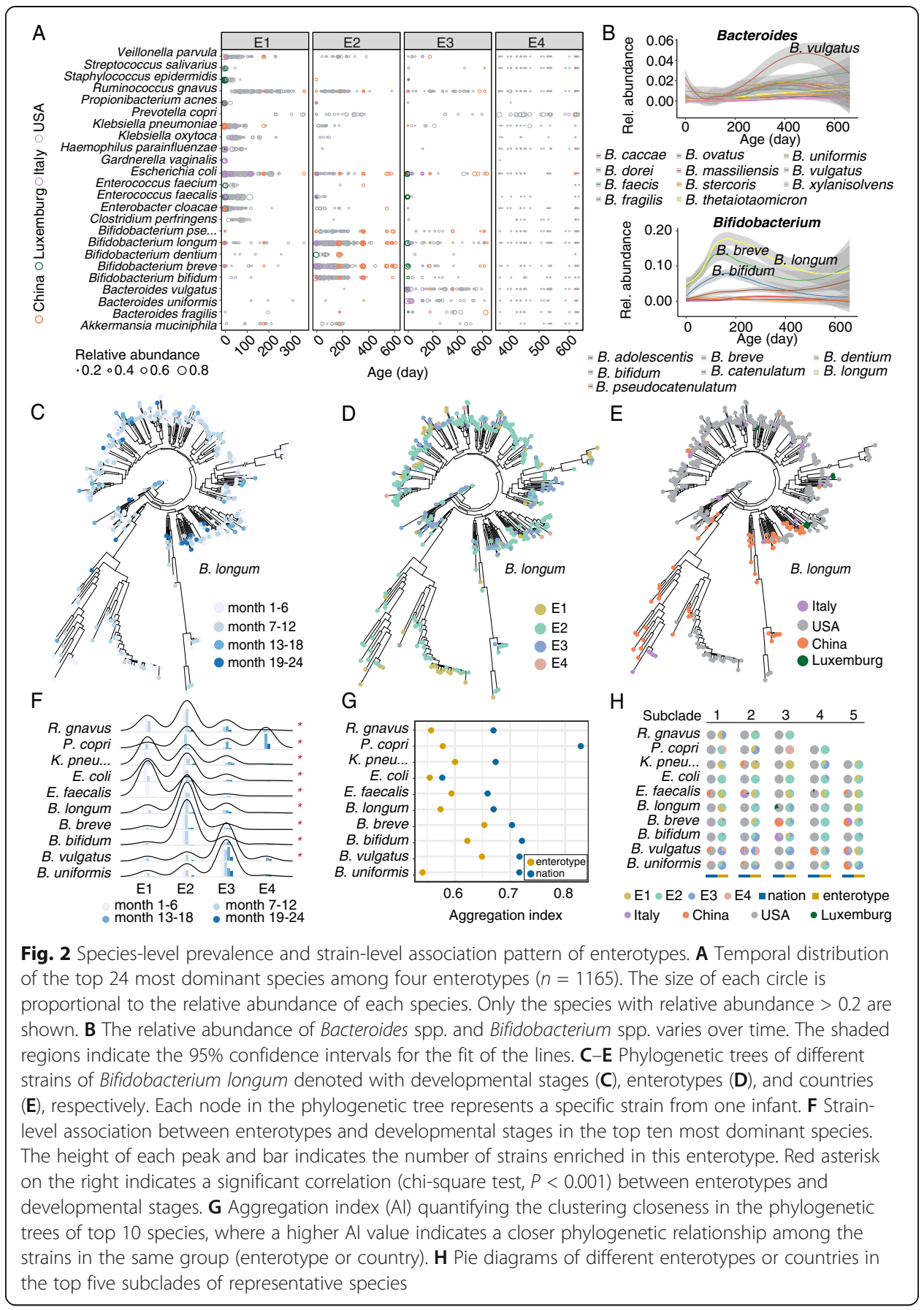

specific species were found and were strongly associated with the main bacterial contributors of each enterotype, although the abundance of these species varied with the chronological age of the infants (Fig. 2B). This result indicates that even at the species level, distinct enterotypes may be clearly distinguished from each other and chronological ages may be an important factor associated with enterotypes in the development of infant gut microbiota.

To unveil the reasons underlying the differentiation of the gut microbiota in infants of different chronological ages and countries, we analyzed the microbiota at the strain 
level. Unique marker genes were extracted from each sample and aligned to construct phylogenetic trees for the top ten most abundant species, and then subclades of each tree were counted (Additional file 1: Fig. S7). As an example, phylogenetic trees of Bifidobacterium longum for diverse developmental stages, enterotypes, and countries are shown (Fig. 2C-E). Notably, strains classified into E1 and E2 were precisely correlated to early developmental stages of the gut microbiota, while those from E3 and E4 were associated with later stages (Fig. $2 \mathrm{C}$, D, chi-square test, $P<0.001$ ). Regarding the geographic environment, the direct correlation was much weaker. Infants who belonged to the same developmental stages tended to share the same enterotype strain, regardless of the country of origin. For strains belonging to the same country, enterotypes varied in parallel with infant developmental stages (Fig. 2C-E). The strong correlation between enterotypes and developmental stages demonstrates that the age factor plays an important role on the stratification of enterotypes. The geographical stratification of enterotypes to some extent reflects the differences of developmental stages of infants in these countries.

To verify the correlation between enterotypes and the development of the gut microbiota, we further determined the enterotype association pattern in other species (Additional file 1: Fig. S7). Consistent with the observation in Bifidobacterium longum, the association between enterotypes and developmental stages was much stronger than that between enterotypes and geographical factors (Fig. 2F, G). Enterotypes dominated by Bacteroides and Prevotella exhibited a more mature pattern than the other two enterotypes, while strains from the same country were classified into different enterotypes due to the differences in the maturity of the gut microbiota (Fig. $2 \mathrm{H}$ ), suggesting that the stratification of enterotypes among different countries can correspond to different developmental stages of the infant gut microbiota.

\section{The developmental process of infant gut microbiota is deterministic and predictable}

To explore the developmental process of gut microbiota in the early stage, we stratified 1336 infants (only infants with more than three time points were included) into nine age groups with 4-month intervals to determine the enterotype transitions across different ages. As shown in Fig. 3A, a common inter-enterotype shift tendency was present throughout the entire period. The most frequent transition was observed in the first year of life. During this period, more than half of the infants (52.17\%) in E1 shifted to E2, and some of the subjects previously belonging to E1 (9.24\%) and E2 (9.12\%) transitioned to E3. This transition, however, decelerated soon afterwards. In the second year, the microbiota became more stable, with over half of the infants $(56 \%)$ without interenterotype variation, and in the third year, the ratio was up to $79.6 \%$.

To quantify the transition of different enterotypes in early life microbiota, we used a Markov chain-based approach to model the enterotype transition probabilities (Fig. 3B). E1 showed a high frequency of transition to other enterotypes, with a relatively higher transition probability to E2 and E3 ( 0.37 and 0.39 , respectively) compared to its self-transition probability $(0.22)$. This may be the major reason for the disappearance of E1 in the following 2 years after birth (Fig. 3A). Further, E2 showed a tendency to transition to E3 (0.38). For this reason, although E2 corresponded to the majority of the infants in the first year of life, starting from the second year, E3 outnumbered E2 and 


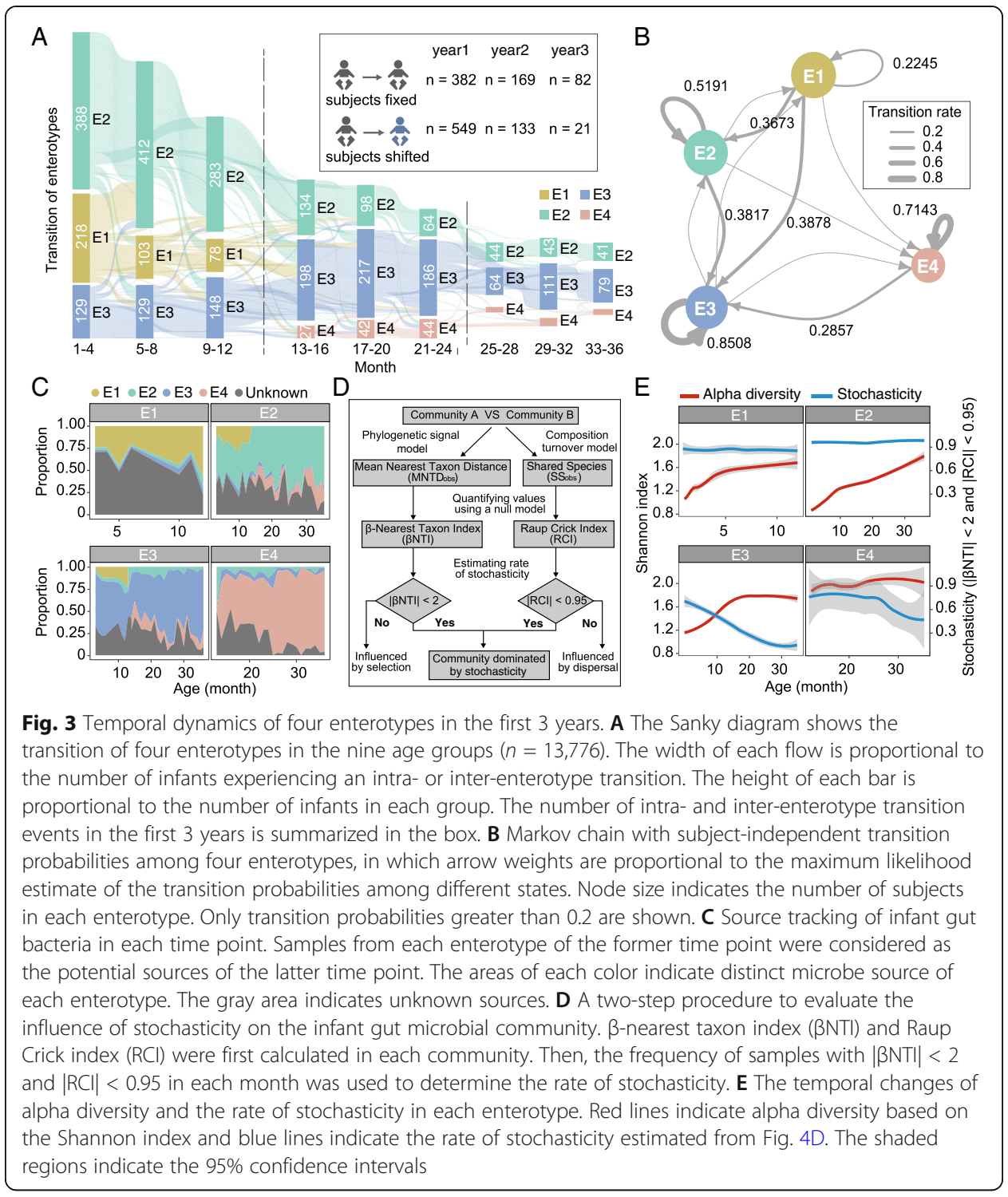

became dominant in infant populations (Fig. 3A). Changes in the abundance of their respective enterotype bacteria also accounted for the decline in E2 and the rise in E3 (Additional file 1: Fig. S5B). To estimate the transition and development of the four enterotypes in the future, we implemented a random forest model with a minimum gap from 30 to 60 days. It was observed that the transition of enterotypes in the early days of life was predictable with an AUC greater than 0.8, and the three enterotype bacteria (Bacteroides, Bifidobacterium, and Prevotella) played a crucial role in this prediction (Additional file 1: Fig. S8A). This result supported our assumption that the transition among enterotypes was a crucial path from stages of immaturity to maturity.

We further tracked the process of enterotype fluctuation by measuring the timeseries changes of gut bacteria in infants, which helped us understand the alternation of microbes during the early development from a precise time scale. Each sample at the former time point served as the potential source to predict the origin of microbes at the latter time point in each enterotype. The results of source tracking exhibited 
distinct patterns in each enterotype (Fig. 3C). We found a remarkably small proportion of microbes from the other three enterotypes imported into E1. In contrast, E3 accepted a large number of bacteria from E1 and E2. This tendency was consistent with the transition of enterotypes and strongly suggested that E1 might be the microbial source of the other enterotypes. Additionally, E3 contained the most microbes from the other enterotypes, thereby indicating its microbial sink status. Although we observed a limited frequency of microbial transmission from E4 to E2 and E3, a high prevalence of self-transmission of microbes in E4 (Fig. 3B, C) enhanced its stable feature compared to $\mathrm{E} 1$ and $\mathrm{E} 2$ and thus implied its maturation.

Since we have demonstrated that the less mature enterotypes (E1, E2) have an apparent tendency to transit to more mature enterotypes (E3, E4), a problem on the understanding of the reason underlying this transition awaits solution. We used an ecological model to explore the internal driving forces of each enterotype to elucidate potential factors affecting the microbiota dynamics (Fig. 4D). As shown in Fig. 3E, in the four enterotypes, the alpha diversity of the gut microbiota was positively correlated with the increasing age of infants, which indicated the gradual maturation of all enterotypes although the pace varied. However, the stochasticity ratios in E1 and E2 $(\sim 90 \%)$ were relatively constant and significantly higher than those in E3 and E4 (16-78\%). Stochasticity ratios dropped sharply with increasing age in E3 (from 75.3 to 16.1\%) and E4 (from 78.3 to $33.3 \%$ ), which implied that these enterotypes were governed by the determined selection and presented a more stable community structure.

We subsequently measured the microbial interactions associated with the four enterotypes to explore the possible causations and trends of enterotype transition. A cooccurrence network at the species level revealed the difference in bacterial interactions (Additional file 1: Fig. S8B) and enhanced the credibility that the community structure of E3 and E4 was much more stable than that of E1 and E2. In these networks, both E1 and E2 presented a weak bacterial interaction, with most of their taxa separated from each other. In E3 and E4, however, the interaction was more frequent and the connection among species was much closer (chi-square test, $P<0.001$ ). Particularly in E4, several species formed a compact Prevotella-centered cluster, which might contribute to the stability of their community structure and promote the maturation of the infant gut microbiota. Interestingly, we also found that the decrease of Bifidobacterium in E2 and the increase of Bacteroides in E3 were associated with the change in abundance of their corresponding bacteriophages (Additional file 1: Fig. S8C), suggesting that bacteriophages might be involved in regulating the abundance of enterotype bacteria during the transition of enterotypes.

\section{Multiple clinical factors are associated with the prevalence of enterotypes}

To identify other factors that might affect microbiota dynamics, we analyzed external clinical factors obtained in this study (Additional file 1: Table S1). Intriguingly, the transition of enterotypes in early life was influenced neither by antepartum (maternal gestation age, the mode of delivery) nor by postpartum factors (the duration of breastfeeding) (Additional file 1: Table S3).

We then implemented PERMANOVA analysis $(n=2287)$ and found that although multiple clinical factors could influence gut microbiota, the effects were much weaker 


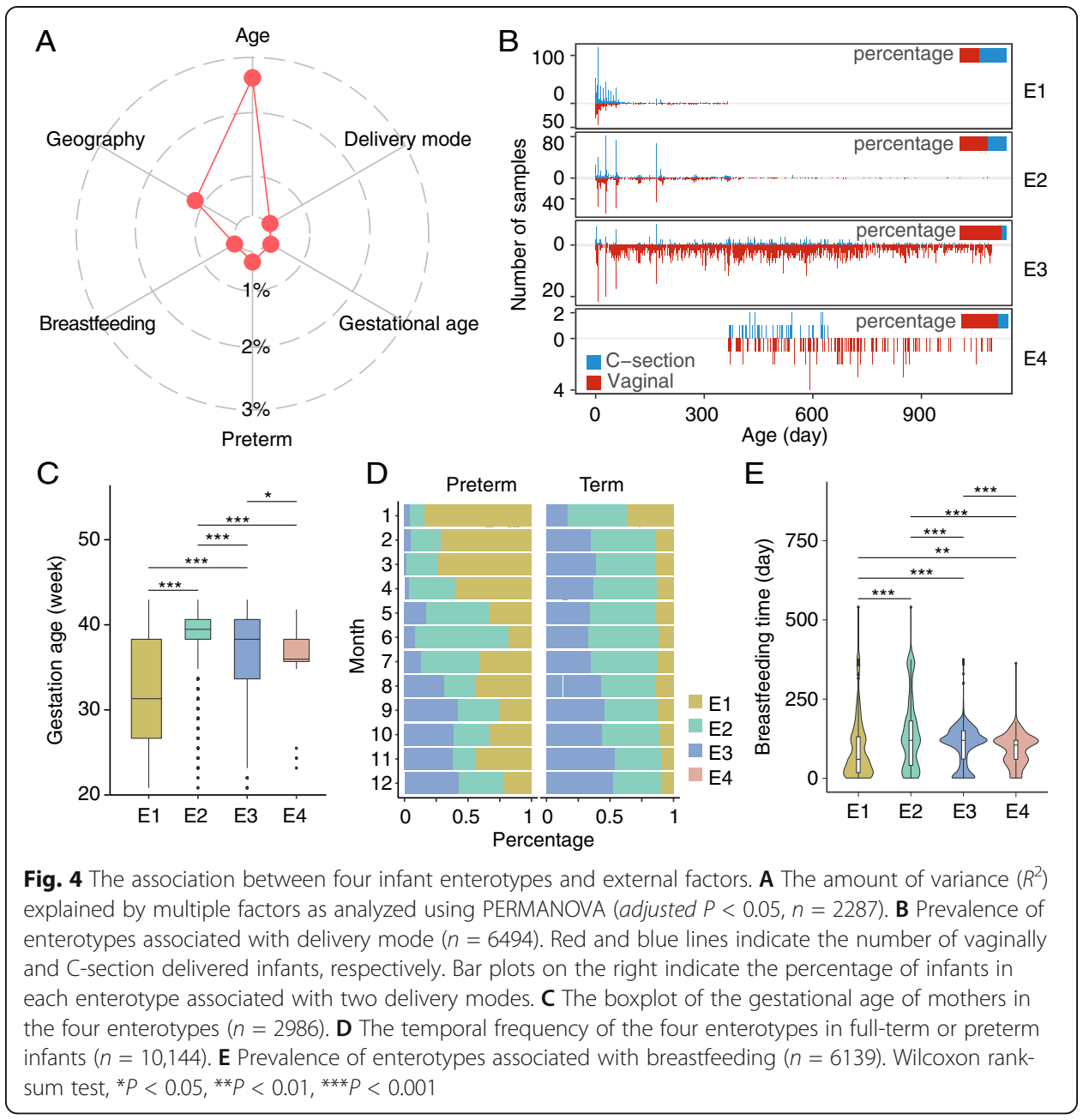

compared with the infant age and the geographical factor (Fig. 4A), indicating that the bacterial community of infant gut experienced a rapid transition in the early age and the developmental stages played an essential role in this process. Considering individual differences may mask the feature of gut microbiota, we then explored the association between clinical factors and enterotypes separately. Among all the infants clustered into $\mathrm{E} 1$, over half were delivered by $\mathrm{C}$-section (59.4\%, hypergeometric test, $P<0.001$ ). In contrast, in the remaining three enterotypes, children delivered via the vaginal route constituted a significant majority (hypergeometric test, $P<0.001$ ) with $59.3 \%$ in E2, 89.6\% in E3, and 78.9\% in E4 (Fig. 4B). Such divergence was also observed at the genus level, in which the gut microbiota of C-section and vaginally delivered infants were dominated by the genera belonging to the phylum Firmicutes and genera Bifidobacterium or Bacteroides, respectively (Additional file 1: Fig. S9A). In addition, infants with E1 showed the lowest gestational age (Wilcoxon test, $P<0.001$ ) (Fig. $4 \mathrm{C}$ ). This tendency was more remarkable in preterm infants (Additional file 1: Fig. S9B), which clustered into E1 2-5 times more than those into E2 and E3 in the first 3 months (Fig. 4D). The correlation was also observed between enterotypes and the duration of breastfeeding; infants who were breastfed for a short ( $<60$ days) or long ( $>300$ days) term tended to be enriched in E1 and E2 (Fig. 4E). Despite the effects of clinical factors exerted on 
gut microbiota were weak (Fig. 4A), there were associations between these factors and the prevalence of enterotypes.

Divergence and transition of the metabolic capacity are associated with the development of infant gut microbiota

To evaluate the potential roles of enterotype differentiation and transition on infant growth and development, we analyzed the functional variations of four enterotypes based on metagenomic data $(n=1165)$. As expected, the four enterotypes grouped into distinct clusters at the functional gene level (Fig. 5A). Consistent with our previous analysis, E3 and E4 were markedly similar in functional gene profiles, although they possessed distinct dominant bacteria. E1 and E2 varied considerably, which suggested that these two enterotypes exhibited distinct functions although they both corresponded to the stage of the very early life.

Furthermore, the metabolic pathways showed marked enterotype- and age-specific patterns (Fig. 5B). A myriad metabolic pathway involved in arginine biosynthesis and branched amino acid biosynthesis were depleted in E1. In contrast, these amino acid biosynthesis pathways were enriched in all age groups of E2. The enriched metabolic pathways in E3 and E4 were similar, most of which were involved in glycolysis, starch degradation, and chorismate, phosphopantothenate, and queuosine biosynthesis.

We then discriminated bacterial contributors to the enriched pathways and found that the enriched amino acid biosynthesis in E2 was largely attributed to the genus Bifidobacterium (Additional file 1: Fig. S10). Additionally, we compared the bacterial taxa with the largest contributions to the functional features of the four enterotypes between the first 2 years (Fig. 5C). Bifidobacterium longum and Bifidobacterium breve accounted for the highest number of significantly enriched metabolic modules in the first year, whereas they were replaced by Bacteroides vulgatus in the second year. This was consistent with the trend observed in the enterotype transition that E2 was initially predominant in infants, but was later exceeded by E3 (Fig. 3A).

It has been demonstrated above that different enterotypes are related to different functions, and as infants grow, E1 and E2 shifted to E3 and E4. We next examined the longitudinal samples of each individual to investigate the association between enterotype transition and metabolic changes. We only chose infants with at least three sampling time points and excluded those without enterotype transition. Among the 257 infants with metagenomic sequencing samples, 119 were retained for downstream analyses. Nearly all these infants showed a dramatic synchronization between the transition of enterotypes and metabolic functions $(t$-test, adjusted $P<0.05)$ (Additional file 1: Fig. S11). For example, in infant TT0132A, the relative abundance of many amino acid biosynthesis pathways (L-arginine and L-isoleucine) and nucleotide biosynthesis (5-aminoimidazole ribonucleotide) decreased sharply when E2 shifted to E3 from day 133 to 205, while the relative abundance of E3-associated pathways (starch degradation and diacylglycerol biosynthesis) exhibited a gradual increase (Fig. 5D).

We finally divided infants who experienced stable transition $(n=61)$ into two groups, group "E1/E2 to E3/E4" ( $n=53)$, which started with E1 or E2 and shifted to E3 or E4 at one stage of growth, and group "E3/E4 to E1/E2" $(n=8)$, which showed an opposite direction in enterotype transition. Among the 32 significantly different pathways 


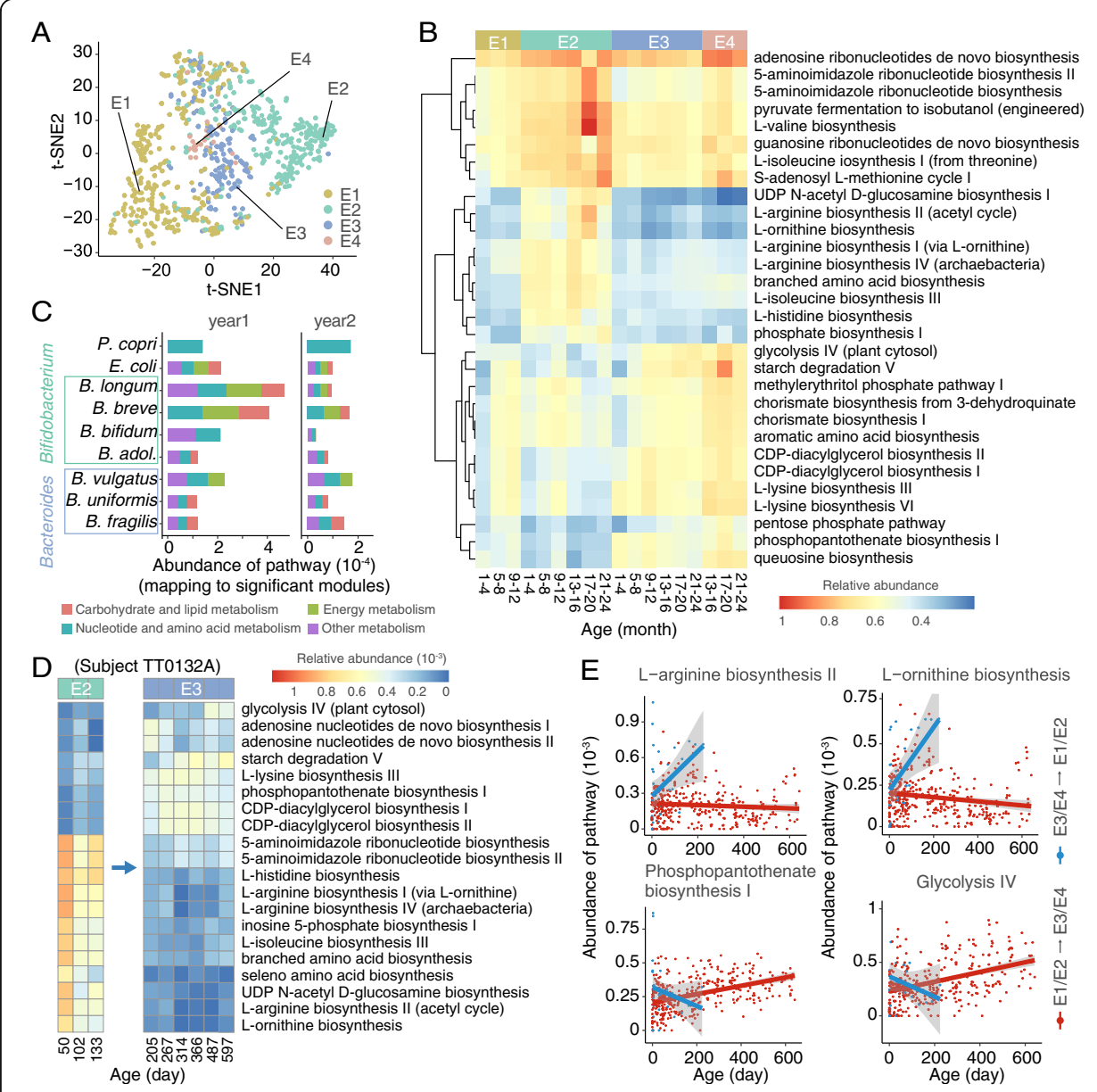

Fig. 5 Divergence and transition of the metabolic capacity associated with the development of the infant gut microbiota. A t-SNE embedding based on Bray-Curtis dissimilarity matrices from the abundance of functional genes $(n=1165)$. B The thirty most significantly differentiated metabolic pathways of four enterotypes in the first 2 years of life. Pathways are hierarchically clustered according to their relative abundance. C Mean abundance of each significantly differentiated module binned at the pathway level. The nine representative species associated with four enterotypes are plotted. D The change of functional features associated with enterotype transition (from E2 to E3) in one American infant (subject TT0132A). The top 20 most differentiated pathways are presented. E An opposite trend over time was found in four representative metabolic pathways between immature (E1/E2) and mature (E3/E4) enterotypes. Infants have been stratified into two subgroups, in which red lines indicate infants with enterotype transition from E1/E2 to E3/E4, and blue lines indicate infants with enterotype transition from E3/E4 to E1/E2. Each dot represents the abundance of the respective pathway from one time point of one infant. The shaded regions indicate the $95 \%$ confidence intervals

between enterotypes (Fig. 5B), fourteen pathways diverged between these two groups (Additional file 1: Fig. S12). For example, L-ornithine biosynthesis and Larginine biosynthesis showed decreased abundance in group "E1/E2 to E3/E4" with increasing age, but increased in the other group. An opposite trend was observed in the metabolic pathways related to phosphopantothenate biosynthesis and glycolysis (Fig. 5E and Additional file 1: Fig. S12). Collectively, these findings indicated that there was a strong correlation between the functional variation and the transition of enterotypes during the development of infants, and the shift from E1 and E2 to E3 and E4 was a common trend not only at the taxonomic but also at the functional level. 


\section{Discussions}

The dynamics of microbial communities in the infant gut have been highly discussed in recent years [2]. In this study, with over 10,000 longitudinal fecal samples of neonates spanning 17 countries, we analyzed the infant gut microbiota with the largest population to date and clustered four robust enterotypes for the first time. The results show that each of these enterotypes was extremely distinct from each other and was not only driven by different bacteria but was also associated with different phases of early development. In addition, a geography-related pattern of enterotypes was observed in the global populations. Our strain-level analysis further indicated that the stratification of enterotypes in different countries can be associated with different developmental stages of the infant gut microbiota. The explicit enterotype transitions and corresponding functional variations observed in this study illustrate the developmental trends of infant gut microbiota from the immature to the mature stages.

The enterotype concept has been debated since it was proposed in 2011 [24]. Many investigations have been performed on adult enterotypes [25, 26], while little has been researched on infants. A major critique raised by researchers is that enterotypes are not discrete states that separate individuals [27]. However, in this study, we observed that the enterotype clustering of infants was much more robust and consistent than that of adults, which might be due to the simplicity of the gut microbiota and limited influencing factors in early age. Apparent barriers among adult enterotypes, especially between Bacteroides- and Prevotella-dominated communities, were observed in previous studies $[25,28]$. Unlike those of adults, infant enterotypes are vulnerable and tend to shift to another type. This inter-enterotype transition may result from the undergoing growth and physiological development of infants as well as external factors such as variable diets $[5,9]$. This frequent transition stabilizes with age, thus denoting the maturation of gut microbiota from infants to adults. Batch effect is inevitable in meta-analyses due to different processing methods in different studies. In our study, however, no studyspecific enterotype was observed during the clustering process, indicating that the enterotype clustering is independent of studies.

The TEDDY study divided the early development of infants into three distinct phases based on the diversity and richness of the gut microbiota. With over ten thousand fecal samples, they emphasized the effect of environmental factors on early development, especially the vital role of breast milk in this process [3]. The findings in our study are basically consistent with such a division that implies frequent fluctuations in the gut microbiota during the first few years of life, while the fluctuation recedes as neonates develop. In addition, the increasing metabolic capacity of amino acid and the importance of Bacteroides in the maturation of the infant gut are also in line with their observations. However, a novel finding in our study is that we observed a geography-related pattern in the stratification of enterotypes, as we have included multi-population cohorts covering 17 countries. Moreover, our study provides a new perspective on the transition of gut microbiota during the early development. This deterministic and predictable transition promotes gut microbiota from immaturity to maturity (Additional file 1: Fig. S13). The gut microbiota of infants in the TEDDY study was characterized with priority colonization of Bifidobacterium, while our study suggests that before the Bifidobacterium-dominant stage, there is an earlier Firmicutes-dominant stage, particularly in preterm infants, which is strongly associated with immaturity. 
We further found that Prevotella was in relatively low abundance in the first few years of life and the Prevotella enterotype did not appear until the second year after birth. This finding was supported by a study that mentioned that the emergence of the Prevotella enterotype occurs much later than that of the Bacteroides enterotype [29]. The anaerobic characteristic and complicated carbohydrate-associated pathway of Prevotella indicate a more mature trait, which may account for the late appearance of this enterotype. Due to its low prevalence (13\% in the second year and $6.2 \%$ the third year) in our study, the Prevotella enterotype was overshadowed by the other three more abundant enterotypes when using the full dataset, in which most samples that should have been classified into the Prevotella enterotype (72.9\%) were falsely assigned to E3 (the Bacteroides enterotype). However, if we clustered enterotypes by sampling the same number of samples from each year, four distinct enterotypes were clearly observed, regardless of the sampling size selected. This clustering result is reproducible when using different clustering methods or even metagenomic datasets. These results emphasize the importance of sampling balance for enterotype clustering. Additionally, previous analyses of enterotypes among school-age children found that the Bifidobacterium enterotype showed the lowest gene number and diversity compared to the other two adult-like enterotypes [30], further demonstrating that the gradual decrease of the Bifidobacterium enterotype was correlated with the development of the infant gut microbiota.

Several studies have emphasized the importance of clinical factors like gestational period and breastfeeding option in early life $[9,31]$. In this study, we confirmed that the maturity of gut microbiota in preterm infants was much delayed compared with that of full-term infants. Only 2287 samples were included in the multivariate analysis due to the lack of information from public studies. Although associations between clinical factors and the prevalence of enterotypes were observed in this study, their effects on gut microbiota were much weaker in the PERMANOVA analysis. We speculated that the divergence between two kinds of analyses may result from the variances of individuals because the geography and the infant age imposed great effects on gut microbiota, which may mask the influences of other factors. In addition, as most of preterm infants were delivered by $\mathrm{C}$-section and fed with breastmilk, the associations we observed in the univariate analysis may be attributed to the influence of prematurity.

Another contribution of this study is that we introduced an ecological model to estimate the tendency of enterotype transitions. Some ecological concepts, such as the $\beta$ mean-nearest taxon distance ( $\beta M N T D)$ and Raup-Crick metric, have been widely applied in many natural ecosystems [32,33]. We adapted this model to the gut microbial community and found that, in the two enterotypes (E1 and E2) in the early stages of life, the rate of stochasticity remained higher during the entire stage of life, while in the other two, it decreased sharply with the increasing age. Given that a high rate of stochasticity in a community indicates its disorder and instability [32], the modeling of stochasticity and selection on different enterotypes explain the trends of shifting from E1 and E2 towards E3 and E4 in the first 3 years of life. Functional features associated with this enterotype shift confirmed that as the infant developed, the four enterotypes displayed a determined metabolic transition, thereby altering the gut microbiota from the composition observed in an immature childhood to that observed in a mature adult-like stage. Nevertheless, research with higher resolution, such as a longer scale 
span and more frequent sampling data, is required to understand the role of gut commensals in different stages of life as well as in different cohorts. Apart from this, the healthy status of the subjects should be associated with different growth patterns to determine outcomes of development in different communities in early life.

\section{Conclusions}

In this study, we presented a comprehensive and quantitative enterotype analysis to elucidate the maturation of gut microbiota from infant to adult-like configuration using 13,776 longitudinal fecal samples from 1965 infants between 1 and 3 years of age, based on multi-population cohorts covering 17 countries. By leveraging the extremely large datasets and enterotype-based microbiome analysis in this study, we deciphered the colonization and transition of the gut microbiota in infants from a new perspective. The four enterotypes were correlated with different developmental stages of infants and exhibited obvious spatial and temporal patterns. We for the first time introduced an ecological model to estimate the tendency of enterotype transitions and demonstrated that the transition of infant gut microbiota was deterministic and predictable.

\section{Materials and methods}

\section{Study cohorts and data retrieval}

A Chinese cohort of newborns was recruited at the Wenzhou People's Hospital, and informed consent was obtained from their parents or guardians. The subject's mother was non-vegetarian and with no antibiotic use during pregnancy and had no history of smoking, alcohol consumption, or any other systemic or metabolic diseases. In total, 101 healthy children born at term were included. All children were of Han ethnicity, and their parents were permanent residents of Wenzhou city, China. Fecal samples were collected at birth, 6, 12, and 18 months of age. Stool was stored in study-provided sterile containers and kept at $-20^{\circ} \mathrm{C}$ and transferred to $-80^{\circ} \mathrm{C}$ upon return to the laboratory. DNA extraction from fecal samples was performed as per methods described previously [34]. The resulting DNA was stored at $-80^{\circ} \mathrm{C}$ until sequencing. For each sample, a random-fragment library (insert length of $\sim 300 \mathrm{bp}$ ) was constructed using the Nextera DNA Sample Preparation Kit (Illumina) with dual indexing and sequenced on the HiSeq 2500 platform (Illumina) to produce 150-bp paired-end reads. Sequencing generated an average of 84.4 million reads per sample, and $88.1 \%$ of the samples had $>10$ million reads. Initial FASTQ files were filtered prior to subsequent analysis using FASTQC. Additionally, sequencing data of 14,821 longitudinal fecal samples were retrieved from 19 public datasets [6, 8-23]. Of these datasets, three cohorts included metagenomic sequencing data and the others were 16S rRNA sequencing data; seven cohorts included preterm infants and twelve cohorts were infants born at term. Children with abnormalities related to growth (such as Down's syndrome, Turner syndrome, Fallot's tetralogy, multiple disabilities, and cystic fibrosis), and children treated with antibiotics during fecal sample collection were excluded. Finally, 1956 healthy children from 23 cities in 17 different countries were included in the study, and their fecal samples covered the first 3 years of life (1-36 months). 


\section{$16 \mathrm{~S}$ rRNA data processing}

For $16 \mathrm{~S}$ rRNA sequencing data profiling, raw reads were analyzed using the opensource software package QIIME [35]. This pipeline selected operational taxonomic units (OTUs) using a reference-based method and then created an OTU table. Briefly, high-quality $16 \mathrm{~S}$ rRNA gene sequences were assigned to OTUs using the script pick closed_reference_otus.py with a $97 \%$ identity threshold. OTUs were subsequently mapped to a subset of the Greengenes database [36]. Abundances were recovered by mapping the demultiplexed reads to the representative OTUs and by producing the final taxonomic profiles. Low-abundance OTUs, whose relative abundance did not reach $0.1 \%$ in at least $10 \%$ of the samples, were excluded.

\section{Metagenomic data processing}

For metagenomic sequencing data profiling, human DNA sequences were identified and removed using KneadData (https://huttenhower.sph.harvard.edu/kneaddata/) by aligning raw reads to the hg19 human reference genome. The adapter and index sequences were trimmed and sequences were quality-filtered using Trimmomatic [37] with the following parameters: -jar trimmomatic-0.36.jar PE -phred33 ILLUMINACLIP: TruSeq3-PE.fa:2:30:10 LEADING:3 TRAILING:3 SLIDINGWINDOW:4:15 HEADCROP:8 MINLEN:36. The relative abundance of bacterial species was calculated using MetaPhlAn [38] with default parameters. Taxonomic tables with relative abundance were merged using the "merge_metaphlan_tables.py" script. The abundance of metabolic pathways was determined using HUMAnN2 [39]. Low-abundance filtering was applied to exclude taxonomic and functional features whose relative abundance did not reach $0.1 \%$ and $0.01 \%$, respectively, in at least $10 \%$ of the samples.

\section{Enterotype clustering}

The enterotype clustering was performed at the genus level according to the previous protocol [24]. The genus with the highest relative abundance in each year was considered as the main contributor of each enterotype. For E1, the main contributors were classified as phylum Firmicutes since the dominant genera possessed a similar relative abundance. To confirm clustering stability, we randomly selected different samples for enterotype clustering and reclassified enterotypes using each cohort. 16S rRNA gene sequencing data and metagenomic sequencing data were also used to reclassify enterotypes, respectively. We further clustered enterotypes by sampling the same number of samples from each year. Regardless of the sampling size selected, they were clearly clustered into four distinct enterotypes. Except for the partitioning around medoid (PAM) method, the Dirichlet multinomial mixtures (DMM) approach was also conducted to verify the clustering results [40].

\section{Microbiota maturation modeling}

Random forest (RF) regression was performed to evaluate the microbiota age as previously described [41]. Briefly, the model was trained on 10\% randomly selected full-term infants ( $>37$ weeks of gestation) belonging to each enterotype in the final dataset. To estimate the minimal number of the top ranked age-discriminatory taxa required for prediction, the rfcv function implemented in the "randomForest" package was applied 
over 100 replicates. This model was then applied to all datasets, and the age of the infants predicted by this model was considered as microbiota age.

\section{Strain-level taxonomic and phylogenetic analysis}

Bacterial strains that were present in multiple samples were identified using StrainPhIAn [42]. The sample-based strain reconstruction and reference databases of each clade and all the reconstructed genomes were analyzed to build multiple sequence alignments and phylogenetic trees.

We used an aggregation index (AI) to quantify the clustering effects through each variance. A cluster was defined as the largest subtree in each phylogeny with all the samples belonging to the same country or enterotype, which contained at least three samples. We defined the aggregation index of a country or enterotype for each species as follows:

$$
\mathrm{AI}=\left[\frac{\left(\sum N_{\mathrm{s}}\right)^{2}}{\left(N_{\mathrm{a}}\right)^{2} \times N_{\mathrm{c}}}\right]^{\frac{1}{10}}
$$

where $N_{\mathrm{a}}$ represents the number of all samples in each phylogenetic tree, $N_{\mathrm{c}}$ the number of clusters in each phylogenetic tree, and $N_{\mathrm{s}}$ the number of samples in each cluster. The AI values increased with the aggregation of clusters in each phylogenetic tree. All phylogenetic trees were visualized using the R package "ggtree".

\section{Tracking the dynamics and bacterial interactions of enterotypes}

All samples were divided into nine groups with 4-month intervals, and the enterotype of each infant was considered to be the most frequent enterotype during each time scale. The inter-enterotype transition rate was quantified and visualized using a Markov chain based on a previously published $\mathrm{R}$ script [43]. Source tracking was applied using the R package "FEAST" [44]. To predict potential sources by month, we only focused on subjects with over three time points in our study. For each month, we randomly selected 100 samples that served as sources and sinks, and ten iterations were performed in this process to obtain average predictions.

\section{Evaluation on the stochasticity of the gut microbiota assembly}

A two-step procedure was performed to estimate the rates of stochasticity in each community [33, 45]. First, the observed degree of phylogenetic turnover of each pairwise community comparison was quantified with the $\beta$-mean-nearest taxon distance ( $\beta$ MNTD) using the $\mathrm{R}$ function "comdistnt" (abundance weighted = TRUE; package "picante") $[46,47]$. The $\beta$ MNTD value quantifies the phylogenetic distance between each OTU in one community $(k)$ and its closest relative in a second community $(m)$ :

$$
\beta M N T D=0.5\left[\sum_{i_{k}=1}^{n_{k}} f_{i_{k}} \min \left(\Delta i_{k} j_{m}\right)+\sum_{i_{m}=1}^{n_{m}} f_{i_{m}} \min \left(\Delta i_{m} j_{k}\right)\right]
$$

where $f_{i_{k}}$ is the relative abundance of OTUi in community $k, n_{k}$ is the number of OTUs in $k$, and $\min \left(\Delta i_{k} j_{m}\right)$ is the minimum phylogenetic distance between OTUi in community $k$ and all OTUsj in community $m$. 
The degree to which $\beta$ MNTD deviates from a null model expectation measures the degree to which the community composition is limited by selection on OTU ecological niches. The difference between the observed $\beta M N T D$ and the mean of the null distribution was measured in units of standard deviation of the null distribution and is referred to as the $\beta$-nearest taxon index ( $\beta \mathrm{NTI})$ :

$$
\beta \mathrm{NTI}=\frac{\beta \mathrm{MNTD}_{\text {obs }}-\overline{\mathrm{\beta MNTD}_{\text {null }}}}{\operatorname{sd}\left(\beta \mathrm{MNTD}_{\text {null }}\right)}
$$

where $\beta M N T D_{\text {obs }}$ is the observed $\beta M N T D, \beta M N T D_{\text {null }}$ is the null value of $\beta M N T D$, and sd indicates the standard deviation of the $\beta M N T D_{\text {null }}$ distribution. We quantified the $\beta$ NTI for all pairwise comparisons using a separate null model for each comparison. $\beta$ NTI values $<-2$ or $>+2$ indicate significantly less than or greater than the expected phylogenetic turnover, respectively. $|\beta \mathrm{NTI}|$ values $<2$ indicate the dominance of stochastic processes in communities [32, 48].

Second, a Raup-Crick metric was calculated to estimate the degree of turnover in OTU composition using R package "vegan" and R code of a previous study [49]. After modification, the Raup-Crick index (RCI) represents the dissimilarity between two communities relative to the null expectation. RCI values between -0.95 and +0.95 denote drift acting alone, which indicates stochastic processes exceeding determined processes in communities [49]. As a result, the rates of stochasticity were recognized from the proportions of community pairs that were between $|\mathrm{RCI}|<0.95$ and $|\beta \mathrm{NTI}|<2$.

\section{Bacteriophage identification and prediction model construction}

We adopted a modified de novo CRISPR pipeline used in a previous study to identify bacteriophages [50,51]. Thirteen enterotype-associated bacteria were first chosen to construct a direct repeat (DR) database. Reference genomes of these bacteria were downloaded from the NCBI database. DRs were identified from bacterial genomes using Piler with default settings [52]. We then extracted the interspaces from our metagenomic reads as CRISPR spacers using CRASS [53]. After comparing with contigs via BLASTn (mismatch $\leq 1$, and $E$-value $\leq 10^{-5}$ ), the target spacers were selected. DRs from the same region were compared with the DR database using BLASTn ( $E$-value $\leq$ $10^{-10}$ and identity $\left.=100 \%\right)$. Significant hits were inferred as the phage source of the spacer (protospacer).

The RF package in $\mathrm{R}$ was used to predict the transition of enterotypes. All subjects sampled less than three time points were discarded. The remaining subjects were randomly divided into an independent training set (90\%) and a testing set (10\%). After tenfold cross-validation, 27 most important features were selected to predict the variation. We applied this model twice with a minimum gap of 30 and 60 days, respectively.

\section{Functional analysis}

Metagenomic data were functionally profiled using HUMAnN2 [39]. Gene family abundance at the community level was calculated to show the contributions of known and unknown bacterial species. The pathway abundance was computed both at the community level and for each species using community- and species-level gene abundances along with the structure of the pathway. Functional clustering was performed based on 
Bray-Curtis dissimilarity matrices of significantly different genes identified through Kruskal-Wallis tests.

\section{Statistical analysis}

A traditional batch-correction method [54] was applied to remove batch effects. The variance explained by the enterotype was much larger than that of the study both before and after correction. All statistical analyses were conducted in $\mathrm{R}$ within RStudio and visualized using package "ggplot2." Because of the lack of sufficient metadata, all samples with clinical information were divided into four discrete groups $(1,6,12,18$ months), and the quantification of the variance was calculated using PERMANOVA as implemented by the "adonis" function in the R package "vegan." Enterotype characteristics and variations associated with clinical factors were compared using chi-square tests for categorical variables and Wilcoxon rank-sum tests for continuous variables. For simple, independent comparisons, $P$-values $<0.05$ were considered significant. For all analyses regarding multiple comparisons, we used the Benjamini-Hochberg method to correct for multiple testing.

\section{Supplementary Information}

The online version contains supplementary material available at https://doi.org/10.1186/s13059-021-02463-3.

Additional file 1: Tables S1-S3, Figures S1-S13. Supplementary tables and figures.

Additional file 2. Reference List of Datasets. Reference list of datasets included in this study.

Additional file 3. Review history

\section{Acknowledgements}

We thank all the participants involved in this study.

Peer review information

Kevin Pang was the primary editor of this article and managed its editorial process and peer review in collaboration with the rest of the editorial team.

Review history

The review history is available as Additional file 3.

Authors' contributions

FZ and JW conceived and designed the study and interpreted the data. JZ and XL collected the samples and conducted the experiments. LX, JW, and FZ analyzed the data and created graphs. LX, JW, and FZ wrote the paper. All authors approved the final version of the manuscript.

Funding

This work was supported by the National Natural Science Foundation of China (32025009, 31722031, 31670119,

31870107, and 32070122) and the Beijing Natural Science Foundation (JQ18020).

Availability of data and materials

Raw sequencing data have been deposited in the Sequence Read Archive database under accession number PRJNA695070 [55]. R scripts and metadata used for data analyses can be accessed at https://zenodo.org/ record/5141515 [56]. Additional file 2 contains a list of previously published datasets used in this study.

\section{Declarations}

Ethics approval and consent to participate

The study protocol was approved by the medical ethics committee of Wenzhou People's Hospital and in accordance with the Helsinki Declaration. Informed consent was obtained from all participants (or the infants' parents).

Consent for publication

Not applicable.

Competing interests

The authors declare that they have no competing interests. 


\section{Author details}

${ }^{1}$ Computational Genomics Laboratory, Beijing Institutes of Life Science, Chinese Academy of Sciences, Beijing 100101, China. 'University of Chinese Academy of Sciences, Beijing, China. ${ }^{3}$ Department of Gynecology and Obstetrics, Wenzhou People's Hospital, Wenzhou 325000, China.

\section{Received: 11 January 2021 Accepted: 5 August 2021}

\section{Published online: 24 August 2021}

\section{References}

1. Robertson RC, Manges AR, Finlay BB, Prendergast AJ. The human microbiome and child growth - first 1000 days and beyond. Trends Microbiol. 2019;27(2):131-47. https://doi.org/10.1016/j.tim.2018.09.008.

2. Derrien M, Alvarez AS, de Vos WM. The gut microbiota in the first decade of life. Trends Microbiol. 2019;27(12):997-1010. https://doi.org/10.1016/j.tim.2019.08.001.

3. Stewart CJ, Ajami NJ, O'Brien JL, Hutchinson DS, Smith DP, Wong MC, et al. Temporal development of the gut microbiome in early childhood from the TEDDY study. Nature. 2018;562(7728):583-8. https://doi.org/10.1038/s41586-01 8-0617-x.

4. Fallani M, Young D, Scott J, Norin E, Amarri S, Adam R, et al. Intestinal microbiota of 6-week-old infants across Europe: geographic influence beyond delivery mode, breast-feeding, and antibiotics. J Pediatr Gastroenterol Nutr. 2010;51(1):7784. https://doi.org/10.1097/MPG.0b013e3181d1b11e.

5. Stearns JC, Zulyniak MA, de Souza RJ, Campbell NC, Fontes M, Shaikh M, et al. Ethnic and diet-related differences in the healthy infant microbiome. Genome Med. 2017;9(1):32. https://doi.org/10.1186/s13073-017-0421-5.

6. Reyman M, van Houten MA, van Baarle D, Bosch A, Man WH, Chu M, et al. Impact of delivery mode-associated gut microbiota dynamics on health in the first year of life. Nat Commun. 2019;10(1):4997. https://doi.org/10.1038/s41467-01 9-13014-7.

7. Kuang YS, Li SH, Guo Y, Lu JH, He JR, Luo BJ, et al. Composition of gut microbiota in infants in China and global comparison. Sci Rep. 2016;6(1):36666. https://doi.org/10.1038/srep36666.

8. Matsuki T, Yahagi K, Mori H, Matsumoto H, Hara T, Tajima S, et al. A key genetic factor for fucosyllactose utilization affects infant gut microbiota development. Nat Commun. 2016;7(1). https://doi.org/10.1038/ncomms11 939.

9. Baumann-Dudenhoeffer AM, D'Souza AW, Tarr PI, Warner BB, Dantas G. Infant diet and maternal gestational weight gain predict early metabolic maturation of gut microbiomes. Nat Med. 2018;24(12):1822-9. https://doi.org/10.1038/s41591-01 8-0216-2.

10. Vatanen T, Plichta DR, Somani J, Munch PC, Arthur TD, Hall AB, et al. Genomic variation and strain-specific functional adaptation in the human gut microbiome during early life. Nat Microbiol. 2019;4(3):470-9. https://doi.org/10.1038/s41 564-018-0321-5.

11. Biagi E, Aceti A, Quercia S, Beghetti I, Rampelli S, Turroni S, et al. Microbial community dynamics in mother's milk and infant's mouth and gut in moderately preterm infants. Front Microbiol. 2018;9:2512. https://doi.org/10.3389/fmicb.2018. 02512 .

12. de Muinck EJ, Trosvik P. Individuality and convergence of the infant gut microbiota during the first year of life. Nat Commun. 2018;9(1):2233. https://doi.org/10.1038/s41467-018-04641-7.

13. Duan M, Yu J, Feng J, He Y, Xiao S, Zhu D, et al. 16 S ribosomal RNA-based gut microbiome composition analysis in infants with breast milk jaundice. Open Life Sci. 2018;13(1):208-16. https://doi.org/10.1515/biol-2018-0025.

14. Ferretti P, Pasolli E, Tett A, Asnicar F, Gorfer V, Fedi S, et al. Mother-to-infant microbial transmission from different body sites shapes the developing infant gut microbiome. Cell Host Microbe. 2018;24:133-145.e135.

15. Gasparrini AJ, Wang B, Sun X, Kennedy EA, Hernandez-Leyva A, Ndao IM, et al. Persistent metagenomic signatures of early-life hospitalization and antibiotic treatment in the infant gut microbiota and resistome. Nat Microbiol. 2019;4(12): 2285-97. https://doi.org/10.1038/s41564-019-0550-2.

16. Gregory KE, Samuel BS, Houghteling P, Shan G, Ausubel FM, Sadreyev RI, et al. Influence of maternal breast milk ingestion on acquisition of the intestinal microbiome in preterm infants. Microbiome. 2016:4(1):68. https://doi.org/10.11 86/s40168-016-0214-X.

17. Hill CJ, Lynch DB, Murphy K, Ulaszewska M, Jeffery IB, O'Shea CA, et al. Evolution of gut microbiota composition from birth to 24 weeks in the INFANTMET Cohort. Microbiome. 2017;5:4. https://doi.org/10.1186/ s40168-016-0213-y.

18. Loughman A, Ponsonby A-L, O'Hely M, Symeonides C, Collier F, Tang MLK, et al. Gut microbiota composition during infancy and subsequent behavioural outcomes. EBioMedicine. 2020;52:102640. https://doi.org/10.1016/j.ebiom.2020.102 640.

19. Raman AS, Gehrig JL, Venkatesh S, Chang HW, Hibberd MC, Subramanian S, et al. A sparse covarying unit that describes healthy and impaired human gut microbiota development. Science. 2019;365(6449):eaau4735. https://doi.org/10.1126/ science.aau4735.

20. Tauchi H, Yahagi K, Yamauchi T, Hara T, Yamaoka R, Tsukuda N, et al. Gut microbiota development of preterm infants hospitalised in intensive care units. Benefic Microbes. 2019;10(6):641-51. https://doi.org/10.3920/BM2019. 0003.

21. Wampach L, Heintz-Buschart A, Fritz JV, Ramiro-Garcia J, Habier J, Herold M, et al. Birth mode is associated with earliest strain-conferred gut microbiome functions and immunostimulatory potential. Nat Commun. 2018;9(1):5091. https://doi. org/10.1038/s41467-018-07631-X.

22. Yuan Z, Yan J, Wen H, Deng X, Li X, Su S. Feeding intolerance alters the gut microbiota of preterm infants. PLoS One. 2019;14(1):e0210609. https://doi.org/10.1371/journal.pone.0210609.

23. Zhou S, Xu R, He F, Zhou J, Wang Y, Zhou J, et al. Diversity of gut microbiota metabolic pathways in 10 pairs of Chinese infant twins. PLoS One. 2016;11(9):e0161627. https://doi.org/10.1371/journal.pone.0161627.

24. Arumugam M, Raes J, Pelletier E, Le Paslier D, Yamada T, Mende DR, et al. Enterotypes of the human gut microbiome. Nature. 2011;473(7346):174-80. https://doi.org/10.1038/nature09944. 
25. Levy R, Magis AT, Earls JC, Manor O, Wilmanski T, Lovejoy J, et al. Longitudinal analysis reveals transition barriers between dominant ecological states in the gut microbiome. Proc Natl Acad Sci U S A. 2020;117(24):13839-45. https:// doi.org/10.1073/pnas.1922498117.

26. Costea Pl, Hildebrand F, Arumugam M, Backhed F, Blaser MJ, Bushman FD, et al. Enterotypes in the landscape of gut microbial community composition. Nat Microbiol. 2018;3(1):8-16. https://doi.org/10.1038/s41564-0170072-8.

27. Knights D, Ward TL, McKinlay CE, Miller H, Gonzalez A, McDonald D, et al. Rethinking "enterotypes". Cell Host Microbe. 2014;16(4):433-7. https://doi.org/10.1016/j.chom.2014.09.013.

28. Roager HM, Licht TR, Poulsen SK, Larsen TM, Bahl MI. Microbial enterotypes, inferred by the Prevotella-to-Bacteroides ratio, remained stable during a 6-month randomized controlled diet intervention with the new nordic diet. Appl Environ Microbiol. 2014;80(3):1142-9. https://doi.org/10.1128/AEM.03549-13.

29. Bergstrom A, Skov TH, Bahl MI, Roager HM, Christensen LB, Ejlerskov KT, et al. Establishment of intestinal microbiota during early life: a longitudinal, explorative study of a large cohort of Danish infants. Appl Environ Microbiol. 2014;80(9): 2889-900. https://doi.org/10.1128/AEM.00342-14.

30. Zhong H, Penders J, Shi Z, Ren H, Cai K, Fang C, et al. Impact of early events and lifestyle on the gut microbiota and metabolic phenotypes in young school-age children. Microbiome. 2019;7(1):2. https://doi.org/10.1186/s40168018-0608-z

31. Chernikova DA, Madan JC, Housman ML, Zain-Ul-Abideen M, Lundgren SN, Morrison HG, et al. The premature infant gut microbiome during the first 6 weeks of life differs based on gestational maturity at birth. Pediatr Res. 2018;84(1):71-9. https://doi.org/10.1038/s41390-018-0022-z.

32. Dini-Andreote F, Stegen JC, van Elsas JD, Salles JF. Disentangling mechanisms that mediate the balance between stochastic and deterministic processes in microbial succession. Proc Natl Acad Sci U S A. 2015;112(11):E1326-32. https:// doi.org/10.1073/pnas.1414261112.

33. Gao C, Montoya L, Xu L, Madera M, Hollingsworth J, Purdom E, et al. Fungal community assembly in drought-stressed sorghum shows stochasticity, selection, and universal ecological dynamics. Nat Commun. 2020;11(1):34. https://doi.org/1 0.1038/s41467-019-13913-9.

34. Wang J, Zheng J, Shi W, Du N, Xu X, Zhang Y, et al. Dysbiosis of maternal and neonatal microbiota associated with gestational diabetes mellitus. Gut. 2018;67(9):1614-25. https://doi.org/10.1136/gutjnl-2018-315988.

35. Caporaso JG, Kuczynski J, Stombaugh J, Bittinger K, Bushman FD, Costello EK, et al. QIIME allows analysis of high-throughput community sequencing data. Nat Methods. 2010;7(5):335-6. https://doi.org/10.1038/nmeth.f.3 03.

36. DeSantis TZ, Hugenholtz P, Larsen N, Rojas M, Brodie EL, Keller K, et al. Greengenes, a chimera-checked 165 rRNA gene database and workbench compatible with ARB. Appl Environ Microbiol. 2006;72(7):5069-72. https://doi.org/10.1128/A EM.03006-05.

37. Bolger AM, Lohse M, Usadel B. Trimmomatic: a flexible trimmer for Illumina sequence data. Bioinformatics. 2014;30(15): 2114-20. https://doi.org/10.1093/bioinformatics/btu170.

38. Truong DT, Franzosa EA, Tickle TL, Scholz M, Weingart G, Pasolli E, et al. MetaPhIAn2 for enhanced metagenomic taxonomic profiling. Nat Methods. 2015;12(10):902-3. https://doi.org/10.1038/nmeth.3589.

39. Franzosa EA, Mclver LJ, Rahnavard G, Thompson LR, Schirmer M, Weingart G, et al. Species-level functional profiling of metagenomes and metatranscriptomes. Nat Methods. 2018;15(11):962-8. https://doi.org/10.1038/s41592-018-0176-y.

40. Holmes I, Quince KHC. Dirichlet multinomial mixtures: generative models for microbial metagenomics. PLoS One. 2012; 7(2):e30126. https://doi.org/10.1371/journal.pone.0030126.

41. Subramanian S, Huq S, Yatsunenko T, Haque R, Mahfuz M, Alam MA, et al. Persistent gut microbiota immaturity in malnourished Bangladeshi children. Nature. 2014;510(7505):417-21. https://doi.org/10.1038/nature13421.

42. Truong DT, Tett A, Pasolli E, Huttenhower C, Segata N. Microbial strain-level population structure and genetic diversity from metagenomes. Genome Res. 2017;27(4):626-38. https://doi.org/10.1101/gr.216242.116.

43. DiGiulio DB, Callahan BJ, McMurdie PJ, Costello EK, Lyell DJ, Robaczewska A, et al. Temporal and spatial variation of the human microbiota during pregnancy. Proc Natl Acad Sci. 2015;112(35):11060-5. https://doi.org/10.1073/pnas.1502 875112.

44. Shenhav L, Thompson M, Joseph TA, Briscoe L, Furman O, Bogumil D, et al. FEAST: fast expectation-maximization for microbial source tracking. Nat Methods. 2019;16(7):627-32. https://doi.org/10.1038/s41592-019-0431-X.

45. Stegen JC, Lin X, Fredrickson JK, Chen X, Kennedy DW, Murray CJ, et al. Quantifying community assembly processes and identifying features that impose them. ISME J. 2013;7(11):2069-79. https://doi.org/10.1038/ismej.2013.93.

46. Webb CO, Ackerly DD, McPeek MA, Donoghue MJ. Phylogenies and community ecology. Annu Rev Ecol Syst. 2002; 33(1):475-505. https://doi.org/10.1146/annurev.ecolsys.33.010802.150448.

47. Fine PVA, Kembel SW. Phylogenetic community structure and phylogenetic turnover across space and edaphic gradients in western Amazonian tree communities. Ecography. 2011;34(4):552-65. https://doi.org/10.1111/j.1600-0587.2 010.06548.x.

48. Stegen JC, Lin X, Konopka AE, Fredrickson JK. Stochastic and deterministic assembly processes in subsurface microbial communities. ISME J. 2012;6(9):1653-64. https://doi.org/10.1038/ismej.2012.22.

49. Chase JM, Kraft NJB, Smith KG, Vellend M, Inouye BD. Using null models to disentangle variation in community dissimilarity from variation in a-diversity. Ecosphere. 2011;2(2):art24. https://doi.org/10.1890/ES1000117.1.

50. Stern A, Mick E, Tirosh I, Sagy O, Sorek R. CRISPR targeting reveals a reservoir of common phages associated with the human gut microbiome. Genome Res. 2012;22(10):1985-94. https://doi.org/10.1101/gr.138297.112.

51. Wang J, Gao Y, Zhao F. Phage-bacteria interaction network in human oral microbiome. Environ Microbiol. 2016;18(7): 2143-58. https://doi.org/10.1111/1462-2920.12923.

52. Edgar RC. PILER-CR: fast and accurate identification of CRISPR repeats. BMC Bioinformatics. 2007;8(1):18. https://doi.org/1 0.1186/1471-2105-8-18.

53. Skennerton CT, Imelfort M, Tyson GW. Crass: identification and reconstruction of CRISPR from unassembled metagenomic data. Nucleic Acids Res. 2013;41(10):e105. https://doi.org/10.1093/nar/gkt183. 
54. Johnson WE, Li C, Rabinovic A. Adjusting batch effects in microarray expression data using empirical Bayes methods. Biostatistics. 2007;8(1):118-27. https://doi.org/10.1093/biostatistics/kxj037.

55. Xiao L, Wang J, Zheng J, Li X, Zhao F: Deterministic transition of enterotypes shapes the infant gut microbiota at an early age. NCBI SRA. BioProject PRJNA695070. 2021. https://www.ncbi.nlm.nih.gov/bioproject/?term=prjna 695070.

56. Xiao L, Wang J, Zheng J, Li X, Zhao F. Deterministic transition of enterotypes shapes the infant gut microbiota at an early age. Zenodo. 2021; https://zenodo.org/record/5141515\#.YRLV7KZKiO0.

\section{Publisher's Note}

Springer Nature remains neutral with regard to jurisdictional claims in published maps and institutional affiliations.

Ready to submit your research? Choose BMC and benefit from:

- fast, convenient online submission

- thorough peer review by experienced researchers in your field

- rapid publication on acceptance

- support for research data, including large and complex data types

- gold Open Access which fosters wider collaboration and increased citations

- maximum visibility for your research: over $100 \mathrm{M}$ website views per year

At $\mathrm{BMC}$, research is always in progress.

Learn more biomedcentral.com/submissions 